\title{
On Nonlocal Mathematical Morphology
}

\author{
Santiago Velasco-Forero ${ }^{1}$ and Jesus Angulo ${ }^{2}$ \\ ${ }^{1}$ ITWM - Fraunhofer Institute, Kaiserslautern, Germany \\ 2 CMM-Centre de Morphologie Mathématique, \\ Mathématiques et Systèmes, MINES ParisTech, France
}

\begin{abstract}
In this paper, nonlocal mathematical morphology operators are introduced as a natural extension of nonlocal-means in the max-plus algebra. Firstly, we show that nonlocal morphology is a particular case of adaptive morphology. Secondly, we present the necessary properties to have algebraic properties on the associated pair of transformations. Finally, we recommend a sparse version to introduce an efficient algorithm that computes these operators in reasonable computational time.
\end{abstract}

\section{Introduction}

Mathematical morphology is an approach to image analysis that characterises an image by transformations with simple geometrical interpretation [23]. The original image, denoted by $\mathbf{I}$, is studied by its interaction with small subsets, named structuring elements (SEs), obtained by convolution in the max-plus algebra [9]. It has been applied successfully to a large number of fields including biomedical microscopy, material science, remote sensing, and medical imaging. The classical approach is characterised by two main properties [21]: (1) SE is fixed, i.e., does not depend on the spatial position at which it is centred; (2) the basic morphological operations are invariant under translation. This idea has been extended to grey scale images, using a complete lattice formulation [23]. This paper deals with a case of adaptive mathematical morphology. Adaptive mathematical morphology refers to morphological filtering techniques that adjust the SE to the local context of the image. The approach in this paper is based on the adaptive morphology framework, but where the local structuring element is "estimated" taking into consideration the whole image. We thus refer it as a "nonlocal" approach, following the terminology initiated in [1]. The term of "nonlocal morphology" has been already considered in previous works. On the one hand, Salembier in [22] proposed a straightforward generalisation of nonlocal means filter to morphological filters. As we will discuss, our starting point is similar, however the proposed non local structuring function as well as the proposed algebraic framework is totally coherent with classical morphological adjunction theory. On the other hand, Ta et al. [25] introduced a formalism of graph-based nonlocal morphology by generalising the PDE of dilation and erosion. The nonlocal PDE is solved using numerical methods which includes nonlocal distances as weights. It is obvious that such PDE-based approach does 
not induces a couple of adjoint dilation and erosion, and consequently their products do not involve openings and closings in the algebraic sense. We begin with a review of the extensive literature on adaptive mathematical morphology in Section 2. Section 3 covers the development of the nonlocal mathematical morphology. We introduce a simple concept of neighbour system for an image, which allow us to have algebraic morphological transformations. Section 4 introduces the idea of sparse nonlocal morphology and efficient implementations. Finally, Section 5 concludes the paper with some examples and relevant conclusions.

\section{Adaptive mathematical morphology}

Basic Definitions. Mathematical morphology considers an image I as a numerical function from the "spatial" space $\mathrm{E}$ to a "spectral" space $\mathbb{F}$. In the case of grey-level images, $\mathrm{E}$ is a subset of the Euclidean $\mathbb{R}^{n}$ or the discrete space $\mathbb{Z}^{n}$ ( $\mathrm{n}=2$ for $2 \mathrm{D}$ images, $\mathrm{n}=3$ for $3 \mathrm{D}$ images), considered as the support space of the image, and $\mathbb{F}$ is a set of grey-levels, corresponding to the space of values of the image. It is assumed that $\mathbb{F}=\overline{\mathbb{R}}=\mathbb{R} \cup\{-\infty,+\infty\}$ or $\overline{\mathbb{Z}}=\mathbb{Z} \cup\{-\infty,+\infty\}$, or more specifically $\mathbb{F}$ is a closed subset of $\mathbb{R}$, for instance $\mathbb{F}=[a, b]$ for $a, b \in \mathbb{R}$. Thus, a grey-level image is represented by a function,

$$
\mathbf{I}:\left\{\begin{array}{l}
\mathrm{E} \rightarrow \mathbb{F} \\
x \mapsto \mathbf{I}(x)
\end{array}\right.
$$

i.e., $\mathbf{I} \in \operatorname{Fun}(\mathrm{E}, \mathbb{F})$, where $\operatorname{Fun}(\mathrm{E}, \mathbb{F})$ denotes the functions from the discrete support $\mathrm{E}$ onto the space of values of the image $\mathbb{F}$. Accordingly, I maps each pixel $x \in \mathrm{E}$ into a grey-level value $t \in \mathbb{F}, \mathbf{I}(x)=t$. Note that $\mathbb{F}$ with the natural order relation $\leq$ is a complete lattice. It is important to remark that if the $\mathbb{F}$ is a complete lattice, then $\operatorname{Fun}(\mathrm{E}, \mathbb{F})$ is a complete lattice too [24]. Morphological operators aim at extracting relevant structures of the image. This is achieved by carrying out an inquest into the image through a set of known shape called structuring element (SE). The two basic words in the mathematical morphology language are erosion and dilation. They are based on the notion of infimum and supremum. For the case of flat structuring element (SE), the flat erosion and dilation operators are defined as follow,

$$
\varepsilon_{\mathrm{SE}}(\mathbf{I})(x)=\bigwedge_{y \in \operatorname{SE}(x)} \mathbf{I}(y) \quad \text { and } \quad \delta_{\mathrm{SE}}(\mathbf{I})(x)=\bigvee_{y \in \hat{\operatorname{SE}}(x)} \mathbf{I}(y), \quad x \in \mathrm{E}
$$

where $\operatorname{SE}(x) \subseteq \mathrm{E}$ denote the spatial neighbourhood induced by the structuring

element SE centred at $x$, and $\hat{\mathrm{SE}}$ is the transposed structuring element (i.e., reflection w.r.t. the origin).

\subsection{Types of adaptivity in mathematical morphology}

The formulation contained in previous subsection is translation invariant in the space and in the intensity, i.e., the same processing in considered for each pixel $x$ 
in the image I. Several ways have been analysed to define local characteristics of the image in order to locally design the SE at each point of the product space $(x \times$ $t) \in \mathrm{E} \times \mathbb{F}$. There are different ways to define a hierarchy of approaches proposed on adaptive morphology. We use the scheme introduced by [21]. According to the adaptivity considered by the construction of the structuring element, we have two main types:

1. Location-adaptive structuring elements (variability on E [2]): The structuring element $\operatorname{SE}(x)$, depends on the location $x$ in the image. It does not depend on the input image $\mathbf{I}(x)$. One of the earliest application that required the use of variable size SEs is the traffic control camera system [5]. This application inspired [5] to consider the perspective effect in the morphological analysis. Vehicles at the bottom of the image are closer and they appear larger than those higher in the camera. Thus, the SE should follow a law of perspective, for instance, vary linearly with its vertical position in the image. Other example is the term "locally adaptable" used in [10], for SEs as disk where the radius depend on the position of the image.

2. Input-adaptive structuring elements (variability on $\mathbb{F}[2]$ ): The shape of the $\operatorname{SE}(x)$ at $x$ depends on the local features of an image $\mathbf{I}$. We denote this kind of structuring element by $\mathrm{SE}_{\mathbf{I}}(x)$. Examples of this type of adaptive are morphological amoebas [16], intrinsic structuring elements [11], region growing structuring element [20] and morphological bilateral filtering [3]. An example of this type of adaptivity is shown in Fig.1.

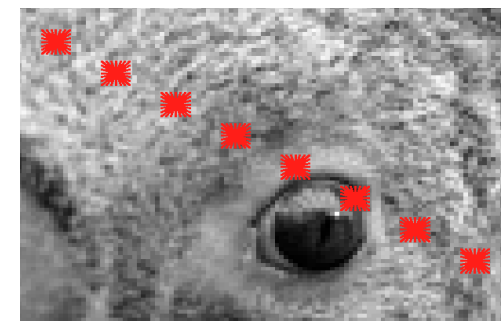

(a) Classical Structuring Elements (b)

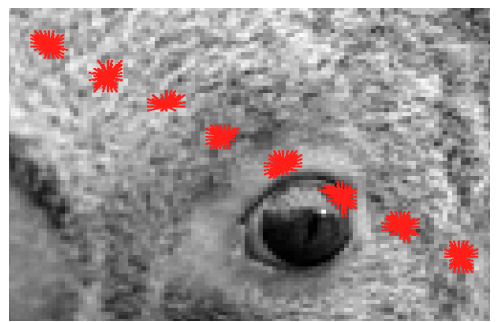

ments

Fig. 1. $S E$ vs $S E_{I}$ for some pixels.

\subsection{Flat Input-Adaptive morphology}

In this subsection, we limit ourselves to the case of flat input-adaptive structuring elements. Let $\mathcal{L}=\operatorname{Fun}(\mathrm{E}, \mathbb{F})$ denote the complete lattice of grey-scale functions with domain $\mathrm{E}$, whose range is a complete lattice $\mathbb{F}$ of grey values. Consider the 
mappings $\delta: \mathcal{L} \rightarrow \mathcal{L}$ and $\varepsilon: \mathcal{L} \rightarrow \mathcal{L}$ defined by:

$$
\delta_{\mathbf{S E}_{\mathbf{I}}}(\mathbf{I})(x):=\bigvee_{y \in \mathrm{SE}_{\mathbf{I}}(x)} \mathbf{I}(y), \text { and } \varepsilon_{\mathbf{S E}_{\mathbf{I}}}(\mathbf{I})(x):=\bigwedge_{y \in \hat{\operatorname{SE}}_{\mathbf{I}}(x)} \mathbf{I}(y), \quad x \in \mathbf{E}
$$

As noted by Roerdink [21], since the neighbourhoods depend on the input I the mappings in (3) are in general not a dilation and erosion, i.e., they do not form an adjunction [14], hence products $\delta \varepsilon$ and $\varepsilon \delta$ are not guaranteed to satisfy the algebraic properties of opening and closing. Additionally, in [21] is given an essential conclusion: "one has to fix the adaptive neighbourhood $\operatorname{SE}_{\mathbf{I}}(x)$ once they have been derived from an initial input image $\mathbf{I}$. The one can apply the operations in (3) to any input image $\mathbf{J}$, and also use combinations of them to construct adaptive opening, closing, alternating sequentiality filters, etc." Thus, in order to have algebraic morphological operators, we need to define a set of adaptive neighbourhoods from a given image $\mathbf{I}$. That is the motivation for the first definition

Definition 1 A structuring elements system on $\mathbf{I}: \mathrm{E} \rightarrow \mathcal{L}$ is a family $\mathrm{SE}_{\mathbf{I}}=$ $\left\{\operatorname{SE}_{\mathbf{I}}(x)\right\}_{x \in \mathrm{E}}$ such that for all $x, y \in \mathrm{E}$,

1. $x \in \mathrm{SE}_{\mathbf{I}}(x)$,

2. $y \in \mathrm{SE}_{\mathbf{I}}(x) \Rightarrow x \in \mathrm{SE}_{\mathbf{I}}(y)$.

The subset $\mathrm{SE}_{\mathbf{I}}(x)$ is called the structuring element of $x$ on the image $\mathbf{I}$.

Note that the structuring element system includes the flat symmetric structuring elements [23], intrinsic structuring elements [11], location-adaptive structuring element [21], and spatially-variant morphology [6]. A fundamental concept in mathematical morphology which plays a role of pseudo-inverse in mathematical morphology, is the adjunction [23]. The adjunction concept associated to adaptive morphology is a misleading concept. See for instance [21] for a pleasant description of this problem. The main advantage of Definition 1 is that allows to formulate the Theorem 1.

Theorem 1. If $\mathrm{SE}_{\mathbf{I}}$ is a structuring element system on $\mathbf{I}$ then $\delta_{\mathrm{SE}_{\mathbf{I}}}\left(\mathbf{J}_{1}\right) \leq \mathbf{J}_{2} \Longleftrightarrow$ $\mathbf{J}_{1} \leq \varepsilon_{\mathbf{S E}_{\mathbf{I}}}\left(\mathbf{J}_{2}\right)$, for all $\mathbf{I}, \mathbf{J}_{1}, \mathbf{J}_{2} \in \mathcal{L}$

Proof. Note that the structuring element system depends only on I. Thus, the proof is straightforward from [21].

Corollary 1. $\gamma_{\mathrm{SE}_{\mathbf{I}}}(\mathbf{J}):=\delta_{\mathrm{SE}_{\mathbf{I}}}\left(\varepsilon_{\mathrm{SE}_{\mathbf{I}}}(\mathbf{J})\right)$ is an opening in the algebraic sense, i.e. $\gamma_{\mathbf{S E}_{\mathbf{I}}}(\mathbf{J}) \leq \mathbf{J}$ and $\gamma_{\mathbf{S E}_{\mathbf{I}}}(\mathbf{J})=\gamma_{\mathbf{S E}_{\mathbf{I}}}\left(\gamma_{\mathbf{S E}_{\mathbf{I}}}(\mathbf{J})\right)$, for all $\mathbf{I}$ and $\mathbf{J}$ in $\mathcal{L}$. Additionally, the dual operator $\varphi_{\mathbf{S E}_{\mathbf{I}}}(\mathbf{J}):=\varepsilon_{\mathbf{S E}_{\mathbf{I}}}\left(\delta_{\mathbf{S E}_{\mathbf{I}}}(\mathbf{J})\right)$ is a closing in the algebraic sense.

Particular cases of this algebraic opening/closing definition can be found in the literature, for instance, Lerallut et al. in [16] proposed the computation of the adaptive structuring element called amoeba from a pilot image, which includes always the central pixel (origin). Adaptive geodesic neighbourhoods in [13] and bilateral flat structuring element [3] uses respectively a threshold over geodesic 
distances or convex combination of spatial distance and a pixel value distance to induce a spatial adaptive structuring element.

Remark 1: Note that $\mathrm{SE}_{\mathbf{I}}$ is fixed. That important issue, illustrated in [21], involves that if $\mathbf{J}=\delta_{\mathbf{S E}_{\mathbf{I}}}(\mathbf{I})$, the operator $\varepsilon_{\mathbf{S E}_{\mathbf{J}}}(\mathbf{J})$ is not an opening in the algebraic sense. In our notation, that means that in general $\varepsilon_{\mathbf{S E}_{\mathbf{J}}}(\mathbf{J}) \neq \gamma_{\mathbf{S E}_{\mathbf{I}}}(\mathbf{I})$. In practice, you cannot apply adaptive dilation followed by adaptive erosion to obtain an adaptive opening in the algebraic sense.

\section{Non-flat nonlocal morphology}

In order to fully understand how and why nonlocal morphology works, we will begin with a detailed description of nonlocal means and the theory which support the approach. Nonlocal processing refers to the general methodology of designing energies using nonlocal comparison of patches extracted in the image. Starting from the initial paper by Baudes et al. [1], nonlocal energies have proved to be efficient for many imaging problems, including denoising [1], semi-supervised classification [12] and segmentation [7]. Recently, nonlocal schemes for image processing have received a lot of attention [8]. Rather than considering only the vector associated to one pixel to compute pixel similarities, patches around these pixels are considered. These patches capture the dependencies of neighbouring pixels and thus can distinguish textural patterns. Nonlocal means filters have been proposed in [8] mainly for denoising applications. The filtering idea consists in computing a weighted average of the input image in a neighbourhood of size $k$ :

$$
\begin{aligned}
\operatorname{NLM}(\mathbf{I}, k)(x) & =\sum_{y \in \mathbf{I}} \mathbf{I}(y) \frac{\widetilde{\mathbf{W}_{\mathbf{I}}}(x, y)}{\sum_{z \in \mathbf{I}} \widetilde{\mathbf{W}_{\mathbf{I}}}(x, z)}, \quad x \in \mathrm{E} \\
& =\sum_{y \in \mathbf{I}} \mathbf{I}(y) \mathbf{W}_{\mathbf{I}}(x, y), \quad x \in \mathbf{E}
\end{aligned}
$$

where the weight $\mathbf{W}_{\mathbf{I}}(x, y)$ is defined by computing the similarity between a patch $\mathbf{P}$ centred around the pixel $x$ and a patch around $y \in \mathbf{S E}_{\mathbf{I}, k}(x)$

$$
\left.\widetilde{\mathbf{W}_{\mathbf{I}}}(x, y):=\widetilde{\mathbf{W}_{\mathbf{I}}}(\mathbf{P}(x), \mathbf{P}(y))\right)=\exp \left(-\frac{\|\mathbf{P}(x)-\mathbf{P}(y)\|^{2}}{\sigma^{2}}\right), \quad x \in \mathrm{E}
$$

Here, $\|\mathbf{P}\|$ is the Euclidean norm of the patch $\mathbf{P}$ of size $l \times l$ as a vector in $\mathbb{R}^{l \times l}$ and $\sigma$ is a smoothing parameter. Thus, pixels with similar neighbourhoods are given larger weights compared to pixels whose neighbourhoods look different. The algorithm makes explicit use of the fact that repetitive patterns appear in most of the natural images. The idea is illustrated in Fig. 2. For a review of the evolution of nonlocal modelling in imaging we recommend [15]. The "natural" morphological extension of the nonlocal means defining (4) is the version on the 


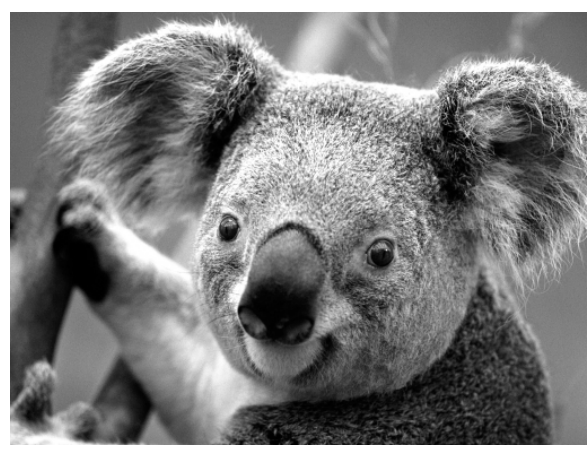

(a) Original image

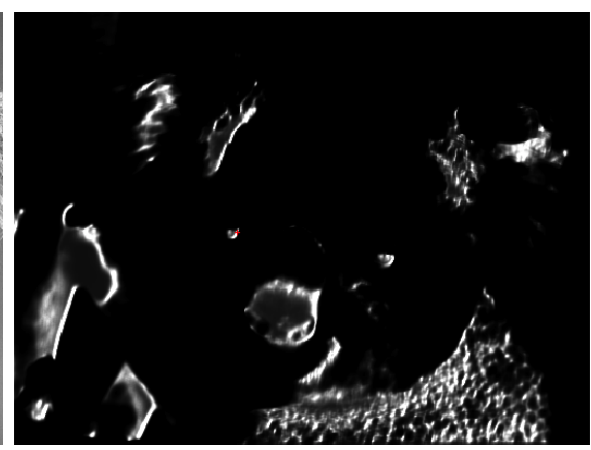

(b) Equation (5) for a pixel centred at the left-eye of the koala.

Fig. 2. Example of nonlocal-functional based on the grey-patch information. The centre pixel is marked by a red-cross. Original image has $384 \times 512$ pixels.

max-plus algebra ${ }^{3}$, which involves replacing the convolution (i.e. $\sum_{y \in \mathbf{I}}$ ) by the supremum or infimum (i.e., $\bigvee_{y \in \mathbf{I}}$ or $\bigwedge_{y \in \mathbf{I}}$ ) and the kernel weights $\mathbf{W}_{\mathbf{I}}$ by their component-wise $\log$ arithm $\mathcal{W}_{\mathbf{I}}=\log \left(\mathbf{W}_{\mathbf{I}}\right)$, i.e., :

$\delta_{\mathrm{SE}_{\mathbf{I}}, \mathcal{W}_{\mathbf{I}}}(\mathbf{I})(x)=\bigvee_{y \in \operatorname{SE}_{\mathbf{I}}(x)}\left(\mathbf{I}(y)+\mathcal{W}_{\mathbf{I}}(x, y)\right)$, and $\varepsilon_{\mathbf{S E}_{\mathbf{I}}, \mathcal{W}_{\mathbf{I}}}(\mathbf{I})(x)=\bigwedge_{y \in \operatorname{SE}_{\mathbf{I}}(x)}\left(\mathbf{I}(y)-\mathcal{W}_{\mathbf{I}}(x, y)\right)$,

for $x \in$ E. A similar expression to (6) was presented by Salembier in [22] without including the logarithmic transformation on $\mathbf{W}_{\mathbf{I}}$. To justify this logarithmic connection between the standard algebra $(+, \times)$ and $(\max ,+)$ algebra underlying morphological operators, the reader is referred to $[18,9,4]$. At this point, a question arise, is the pair $\left(\varepsilon_{\mathbf{S E}_{\mathbf{I}}, \mathcal{W}_{\mathbf{I}}}(\mathbf{I}), \delta_{\mathbf{S E}_{\mathbf{I}}, \mathcal{W}_{\mathbf{I}}}(\mathbf{I})\right)$ an adjunction in the algebraic sense?

Definition 2 A morphological weight system $\mathcal{W}_{\mathbf{I}}: \mathrm{E} \times \mathrm{E} \mapsto \mathbb{R}^{+}$on $\mathbf{I}$ is a weight function such for all $x, y \in \mathrm{E}$,

1. $\mathcal{W}_{\mathbf{I}}(x, x)=0 \quad \forall x \in \mathrm{E}$,

2. $\mathcal{W}_{\mathbf{I}}(x, y)=\mathcal{W}_{\mathbf{I}}(y, x) \quad \forall x, y \in \mathrm{E}$,

3. $-\infty \leq \mathcal{W}_{\mathbf{I}}(x, y) \leq 0 \quad \forall x, y \in \mathrm{E}$.

In fact, we note that all conditions in Definition 2 are valid for the nonlocal weights in (4) due to the facts that $0 \leq \mathbf{W}_{\mathbf{I}} \leq 1$ and $\mathbf{W}_{\mathbf{I}}$ is a 1-diagonal and symmetric matrix.

Theorem 2. If $\mathrm{SE}_{\mathbf{I}}$ is a structuring element system and $\mathcal{W}_{\mathbf{I}}$ a weight system on I then $\delta_{\mathrm{SE}_{\mathbf{I}}, \mathcal{W}_{\mathbf{I}}}\left(\mathbf{J}_{1}\right) \leq \mathbf{J}_{2} \Longleftrightarrow \mathbf{J}_{1} \leq \varepsilon_{\mathrm{SE}_{\mathbf{I}}, \mathcal{W}_{\mathbf{I}}}\left(\mathbf{J}_{2}\right)$, for all $\mathbf{I}, \mathbf{J}_{1}, \mathbf{J}_{2} \in \mathcal{L}$

\footnotetext{
${ }^{3}$ A max-plus algebra is a semiring over the union of real numbers and $-\infty$, equipped with maximum and addition as the two binary operations instead of + and $\times$ operators as in standard algebra.
} 
Proof. Firstly, note that the structuring elements system depends only on $\mathbf{I}$. The proof is straightforward from [21], however it is included to make this article globally self-contained, and then more comprehensible for the reader.

$$
\begin{aligned}
& \delta_{\mathrm{SE}_{\mathbf{I}}, \mathcal{W}_{\mathbf{I}}}\left(\mathbf{J}_{1}\right) \leq \mathbf{J}_{2} \Longleftrightarrow \delta_{\mathbf{S E}_{\mathbf{I}}, \mathcal{W}_{\mathbf{I}}}\left(\mathbf{J}_{1}\right)(x) \leq \mathbf{J}_{2}(x), \forall x \in \mathrm{E} \\
& \Longleftrightarrow \bigvee_{y \in \mathrm{SE}_{\mathbf{I}}(x)} \mathbf{J}_{1}(y)+\log \left(\mathcal{W}_{\mathbf{I}}(x, y)\right) \leq \mathbf{J}_{2}(x), \forall x \in \mathrm{E} \\
& \Longleftrightarrow \mathbf{J}_{1}(y)+\log \left(\mathcal{W}_{\mathbf{I}}(x, y)\right) \leq \mathbf{J}_{2}(x), \forall x \in \mathrm{E}, \forall y \in \mathrm{SE}_{\mathbf{I}, k}(x) \\
& \Longleftrightarrow \mathbf{J}_{1}(y) \leq \mathbf{J}_{2}(x)-\log \left(\mathcal{W}_{\mathbf{I}}(x, y)\right), \forall y \in \mathrm{E}, \forall x \in \mathrm{SE}_{\mathbf{I}, k}(y) \\
& \Longleftrightarrow \mathbf{J}_{1}(y) \leq \bigwedge_{x \in \mathrm{SE}_{\mathbf{I}}(y)} \mathbf{J}_{2}(x)-\log \left(\mathcal{W}_{\mathbf{I}}(x, y)\right), \forall y \in \mathrm{E} \\
& \Longleftrightarrow \mathbf{J}_{1}(y) \leq \bigwedge_{x \in \mathrm{SE}_{\mathbf{I}}(y)} \mathbf{J}_{2}(x)-\log \left(\mathcal{W}_{\mathbf{I}}(y, x)\right), \forall y \in \mathrm{E} \\
& \Longleftrightarrow \mathbf{J}_{1}(y) \leq \varepsilon_{\mathbf{S E}_{\mathbf{I}}, \mathcal{W}_{\mathbf{I}}}(\mathbf{I})(y), \forall y \in \mathrm{E} \\
& \Longleftrightarrow \mathbf{J}_{1} \leq \varepsilon_{\mathbf{S E}_{\mathbf{I}}, \mathcal{W}_{\mathbf{I}}(\mathbf{I}), \forall y \in \mathrm{E}}
\end{aligned}
$$

by max and $\log -\infty$ and 0 .

by 2 in Definition 2

by min and $\log$

by 3 in Definition 2

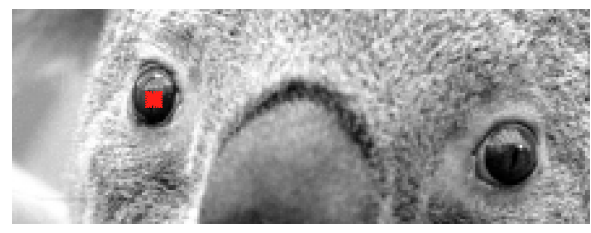

(a) Local structuring element.

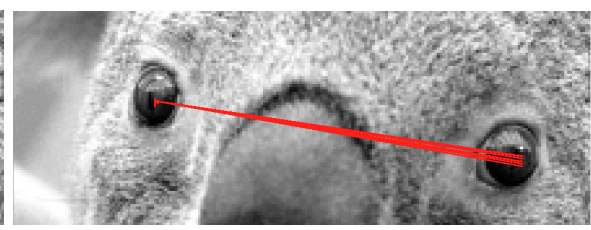

(b) Sparse nonlocal structuring element.

Fig. 3. Local $(\mathrm{SE}(x))$ vs nonlocal structuring element $\left(\mathrm{SE}_{k, \mathbf{I}}(x)\right)$ for the some pixels.

Corollary 2. $\gamma_{\mathrm{SE}_{\mathbf{I}}, \mathcal{W}_{\mathbf{I}}}(\mathbf{J}):=\delta_{\mathrm{SE}_{\mathbf{I}}, \mathcal{W}_{\mathbf{I}}}\left(\varepsilon_{\mathrm{SE}_{\mathbf{I}}, \mathcal{W}_{\mathbf{I}}}(\mathbf{J})\right)$ is an opening in the algebraic sense, i.e., $\gamma_{\mathbf{S E}_{\mathbf{I}}}(\mathbf{J}) \leq \mathbf{J}$ and $\gamma_{\mathrm{SE}_{\mathbf{I}}, \mathcal{W}_{\mathbf{I}}}(\mathbf{J})=\gamma_{\mathrm{SE}_{\mathbf{I}}, \mathcal{W}_{\mathbf{I}}}\left(\gamma_{\mathbf{S E}_{\mathbf{I}}, \mathcal{W}_{\mathbf{I}}}(\mathbf{J})\right)$, for all $\mathbf{I}$ and $\mathbf{J}$ in $\mathcal{L}$. Additionally, the dual operator, $\varphi_{\mathrm{SE}_{\mathbf{I}}, \mathcal{W}_{\mathbf{I}}}(\mathbf{J}):=\varepsilon_{\mathbf{S E}_{\mathbf{I}}, \mathcal{W}_{\mathbf{I}}}\left(\delta_{\mathrm{SE}_{\mathbf{I}}, \mathcal{W}_{\mathbf{I}}}(\mathbf{J})\right)$ is a closing in the algebraic sense.

Definition 3 The matrix representation $\mathcal{W}$ of a morphological weight system $\mathcal{W}_{\mathbf{I}}$ given an image $\mathbf{I}$ with $n$ pixels $x_{1}, x_{2}, \ldots, x_{n} \in \mathrm{E}$ is the square matrix of size $n \times n$ defined by $\mathcal{W}=\left[\mathcal{W}_{\mathbf{I}}\left(x_{i}, x_{j}\right)\right]=\left[\log \mathbf{W}_{\mathbf{I}}\left(x_{i}, x_{j}\right)\right], \forall i, j=1, \ldots, n$.

Remark 2: From Definition 2, it is easy to see that $\mathcal{W}$ in Definition 3 should be symmetric and with diagonal equal to zero. Additionally, $\mathbf{W}$ is not forced to be positive semi-definitive as in most of the linear kernel based filtering [19]. However, any positive definitive kernel induce a weight system in E. In this section it was shown how nonlocal morphology is a particle case of adaptive morphology and a relevant conclusion was presented about this misleading term. However, 
the implementation of this approach requires the computation of a max-plus convolution with a full matrix $\mathcal{W}$ which in computationally intractable. We propose a solution to this bottleneck by modifying the neighbourhood connectivity mapping to connect only a small number of neighbours. Thus, it is possible to implement with almost linear complexity, as it is presented in the next section.

\section{Sparse nonlocal morphology}

In the original formulation of nonlocal morphology in [22], dilation and erosion are analysed by incorporating only the information from the $k$-nearest neighbours ( $k \mathrm{NNs}$ ) according to the patch distance in (5). We denote this as $\mathrm{SE}_{\mathbf{I}, k}$. By simply plug-in $\mathrm{SE}_{\mathbf{I}, k}$ in (6), we obtain:

$$
\delta_{\mathrm{SE}_{\mathbf{I}, k}, \mathcal{W}_{\mathbf{I}}}(\mathbf{I})(x)=\bigvee_{y \in \mathrm{SE}_{\mathbf{I}, k}(x)}\left(\mathbf{I}(y)+\left(\mathcal{W}_{\mathbf{I}}(x, y)\right)\right), \quad x \in \mathrm{E}
$$

It is important to note that the proposal in [22] fails to identify the importance issue of Properties 2 and 3 in Definition 2, i.e., the symmetry of $\mathcal{W}_{\mathbf{I}}$ and logarithmic relationship between $\mathcal{W}_{\mathbf{I}}$ and $\mathbf{W}_{\mathbf{I}}$. Note that the $k \mathrm{NN}$ is not a reflexive relation, i.e., given a set of vectors $\mathcal{X}=\left\{\mathbf{x}_{1}, \mathbf{x}_{2}, \ldots, \mathbf{x}_{n}\right\}$, if $\mathbf{x}_{1}$ is a $k \mathrm{NN}$ of $\mathbf{x}_{2}$ on $\mathcal{X}$ does not imply that $\mathbf{x}_{2}$ is a $k$-nearest neighbours of $\mathbf{x}_{1}$ on $\mathcal{X}$. So, the structuring element system $\mathbf{S E}_{\mathbf{I}, k}$ does not follow the Property 2 in the Definition 1 . To have the symmetric property, a simple approach is to define the $\mathbf{x}_{i}$ as a $k$-NN of $\mathbf{x}_{j}$ based on the metric $d$ if $d\left(\mathbf{x}_{i}, \mathbf{x}_{j}\right)$ is among the $k$ smallest elements of the set $\left\{d\left(\mathbf{x}_{i}, \mathbf{x}_{j}\right) \mid j=1, \ldots, i-1, i+1, \ldots, n\right\}$ or viceversa. A illustrative example of sparse structuring element is show in Fig 3. Implementation. A large part of the success of mathematical morphology in the imaging engineering community is due to the algorithmic developments. Very efficient algorithms have been proposed for translation invariant morphological operators for both binary and grey scale images. However, algorithms addressing the case of adaptive $\mathrm{SE}_{\mathbf{I}}$ are still very limited. Here, we proposed an efficient implementation for the case of sparse nonlocal morphology ( $\left.\mathrm{SE}_{\mathbf{I}}\right)$ based on sparse matrices. Basically, we solve the matrix product directly in the algebra $(\max ,+)$ taking advantage of the sparsity of the structuring element system. So, we define a square matrix of size $n \times n$ denoted by $\dot{\mathbf{W}}_{\mathrm{SE}_{\mathbf{I}, k}}$ or by abuse of notation $\mathbf{W}=[\mathbf{W}(i, j)]$ if $j \in \mathrm{SE}_{\mathbf{I}, k}(i)$ and 0 otherwise. Thus, an adaptive dilation (erosion) may be solved efficiently as it is presented in Algorithm 1. In sparse matrices only the non-zero entries are stored. We denote as findnonzero( $\mathbf{W})$ the function to obtain the non-zero entries of a sparse matrix $\mathbf{W}$. Each entry in the output represents an element $\mathbf{w}_{i, j}$ of the matrix and can be accessed by the two indices $i$ and $j$. Accordingly, $\max \cdot \operatorname{row}(\mathbf{X})$ denotes the vector of the maximum of each row of $\mathbf{X}$, and $\operatorname{vec}(\mathbf{X})$ the vectorization of $\mathbf{X}$, i.e., the linear transformation to convert the matrix into a column vector.

Theorem 3. Every adaptive dilation(erosion) based on a sparse matrix $\dot{\mathbf{W}}$ can be computed in time $O(n k \log (k))$ and space $O(n k)$, where $n$ is the number of pixels of the image and $k>0$. 


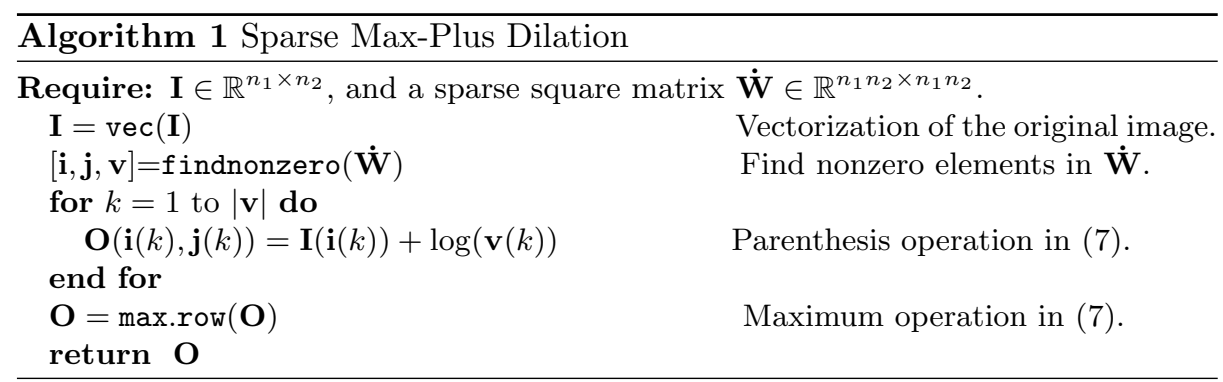

Proof. To compute a dilation, we multiply $n$ times the original image $\mathbf{I}$ as a vector to each of the rows of the sparse matrix $\dot{\mathbf{W}}$ of size $n \times n$ with $k$ values different of zeros $(O(k n))$. A maximum operation should computed in rows, i.e., $O(k \log (k) n)$. Thus, the complexity of Algorithm 1 is $O(k \log (k) n)$. However, note that usually $k \ll n$ then the computation time tends to be linear $O(n)$.

Connections to graph theory. To warm up, let us start by recalling some graph-theoretic definitions.

- A graph $\mathcal{G}$ is pair of sets $\mathcal{G}=(\mathcal{V}, \mathcal{E})$, where the elements of $\mathcal{E}$, called edges, are unordered pairs of elements from $\mathcal{V}$, called vertices.

- A sequence $x_{1}, x_{2}, \ldots, x_{k}$ of distinct vertices of a graph $\mathcal{G}=(\mathcal{V}, \mathcal{E})$ is called a path between $x_{1}$ and $x_{k}$ if $\left\{x_{i}, x_{i+1}\right\} \in \mathcal{E}$ whenever $0 \leq i<k$. The length of the path is $k$, which is the number of edges in the path.

- A graph $\mathcal{G}=(\mathcal{V}, \mathcal{E})$ is said to be connected if there is a path between every pair of vertices in $\mathcal{V}$.

- The adjacency matrix $\mathbf{A}_{\mathcal{G}}$ of a graph $\mathcal{G}$ with $n$ vertices is a $n \times n$ matrix $\mathbf{A}_{\mathcal{G}}=\left(a_{i j}\right)$ in which the entry $a_{i j}=1$ if there is an edge from the vertex $i$ to vertex $j$ and is 0 if there is no edge from vertex $i$ to vertex $j$.

The follows definitions are valid for connected graphs.

- The distance $d(x, y)$ between a pair of vertices $x, y \in \mathcal{V}$ is the length of the shortest path between these vertices.

- The eccentricity $e(x)$ of a vertex $x$ is the maximum distance from $x$ to any other vertex, i.e. $e(x)=\max _{y \in \mathcal{V}} d(x, y)$.

- The maximum eccentricity among all vertices of a graph $\mathcal{G}=(\mathcal{V}, \mathcal{E})$ is called the diameter, i.e. $\operatorname{diam}(\mathcal{G})=\max _{x \in \mathcal{V}} e(x)$

- Given a set of data points $\mathbf{X}=\left\{\mathbf{x}_{1}, \mathbf{x}_{2}, \ldots, \mathbf{x}_{n}\right\}$ with $\mathbf{x}_{i} \in \mathbb{R}^{d} \cdot \mathcal{G}_{k}(\mathbf{X})=$ $(\mathcal{V}, \mathcal{E})$ is a directed graph, where $\mathcal{V}=\mathbf{X}$, and the vertex $\left\langle\mathbf{x}_{i}, \mathbf{x}_{j}\right\rangle \in \mathcal{E}$ if and only if $d\left(\mathbf{x}_{i}, \mathbf{x}_{j}\right)$ is among the $k$ smallest elements of the set $\left\{d\left(\mathbf{x}_{i}, \mathbf{x}_{j}\right) \mid j=\right.$ $1, \ldots, i-1, i+1, \ldots, n\}$ or viceversa, where $d$ is a metric.

For a disconnected graph $\mathcal{G}$, the $\operatorname{diam}(\mathcal{G})$ is defined to be the diameter of the largest connected component in $\mathcal{G}$. From a digital image $\mathbf{I}$, we define $\mathcal{G}=(\mathcal{V}, \mathcal{E})$ as an undirected graph with vertex set $\mathcal{V}$ matching the image pixels and edge set $\mathcal{E}$ consisting of unordered pairs of vertices indicating the adjacency between 
the image pixels according to the adaptive structuring element $\mathrm{SE}_{\mathbf{I}}$ (or $\mathbf{S E}_{\mathbf{I}, k}$ for sparse nonlocal morphology). As the graph only depend on $\mathbf{I}$ and $\mathbf{S E}_{\mathbf{I}}$, we use the notation $\mathcal{G}\left(\mathrm{SE}_{\mathbf{I}}\right)$ (or $\mathcal{G}\left(\mathrm{SE}_{\mathbf{I}, k}\right)$ ). Some links between the nonlocal formulation and classical graph theory are easily perceived.

- $\mathcal{G}\left(\mathrm{SE}_{\mathbf{I}, k}\right)$ is a $\mathcal{G}_{k}\left(\mathbf{P}_{\mathbf{I}}\right)$, by the metric (5), where $\mathbf{P}_{\mathbf{I}}$ denotes the patch information of the image $\mathbf{I}$.

- The element-wise product between $\mathbf{A}_{\mathcal{G}\left(\mathbf{S E}_{\mathbf{I}, k}\right)}$ and $\mathbf{W}_{\mathbf{I}}$ is exactly the sparse matrix $\dot{\mathbf{W}}_{\mathrm{SE}_{\mathbf{I}, k}}$ used in Algorithm 1 .

Finally, we enunciate a less intuitive link between morphological operators and graph properties (proof is not included because of space constrains).

Theorem 4. $\delta_{\mathrm{SE}_{\mathbf{I}}}^{i+1}(\mathbf{I})=\delta_{\mathrm{SE}_{\mathbf{I}}}^{i}(\mathbf{I})$ for $i \geq \operatorname{diam}\left(\mathcal{G}\left(\mathrm{SE}_{\mathbf{I}}\right)\right)$.

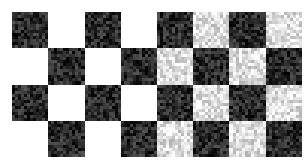

(a) $\mathbf{I}$

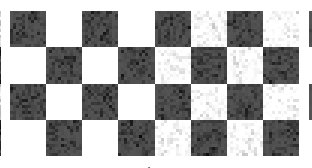

(b) $\delta_{\mathrm{SE}_{I, k}}^{1}(\mathbf{I})$

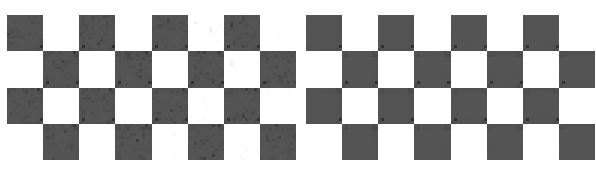

(c) $\delta_{\mathrm{SE}_{\mathbf{I}, k}}^{2}(\mathbf{I})$ (d) $\delta_{\mathrm{SE}_{\mathbf{I}, k}}^{5}(\mathbf{I})$

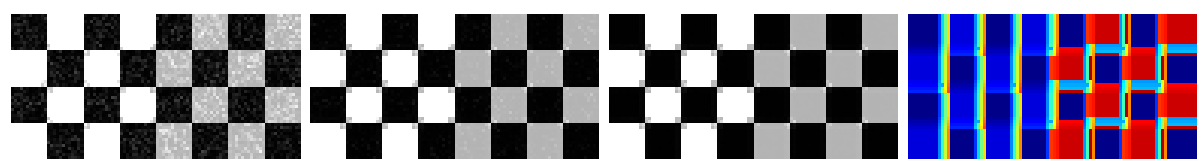
(e) $\varepsilon_{\mathrm{SE}_{\mathbf{I}, k}}^{1}(\mathbf{I})$
(f) $\varepsilon_{\mathrm{SE}_{\mathbf{I}, k}}^{2}(\mathbf{I})$
(g) $\varepsilon_{\mathrm{SE}_{\mathbf{I}, k}}^{5}(\mathbf{I})$
(h) $\mathrm{CC}$ of $\mathcal{G}\left(\mathrm{SE}_{\mathbf{I}, k}\right)$

Fig. 4. Half-chessboard pattern example is a $48 \times 96$ binary image where each square has 144 pixels. The original image (a) is corrupted by impulse noise $(\sigma=.3)$. Flat nonlocal morphology operators, where patches are square $3 \times 3$, and $k=5$ are illustrated in (b)-(g). CC in (h) denotes connected components.

\section{Experiments and Conclusions}

To illustrate the effect of nonlocal morphological operators, we firstly analyse the simple geometrical case of Fig. 4. Nonlocal morphological operators perform quite well due to the connected components of $\mathcal{G}\left(\mathrm{SE}_{\mathbf{I}, k}\right)$, displayed in Fig.4(h), are coherent with the geometric structures of the original image. In the second example, given in Fig. 5, parameters are set to have an unique connected component. We can see that the simplification by nonlocal morphology affects only flat zones of the image, in comparison with classical morphology. However, it is important to remark that the important geometrical interpretation of the classical morphological operators is missing in the nonlocal case. Finally, visual comparison between local and nonlocal dilations and erosions can be performed in a complex image depicted in Fig. 6. To summarise, we studied a class of morphological filters which operate based on patch distance information. We also analysed in detail the requirements to have genuine adaptive morphological 
transformations and, as conclusion, the symmetry and logarithmic connection turns out to be the most relevant properties. Finally, we provided a fast implementation in the case of sparse nonlocal morphology which can be used in any adaptive morphology. Future work includes the comparison of our approach with nonlocal total ordering by manifold learning introduced by [17].

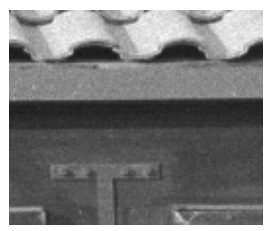

(a) $\mathbf{I}$

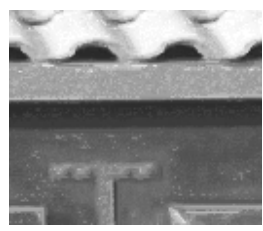

(b) $\delta_{\mathrm{SE}_{\mathbf{I}, k}}^{3}(\mathbf{I})$

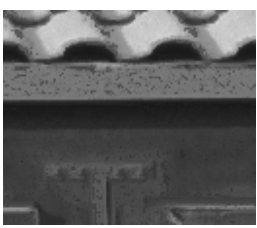

(e) $\varepsilon_{\mathrm{SE}_{\mathbf{I}, k}}^{3}(\mathbf{I})$

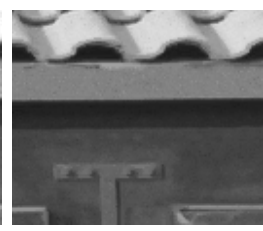

(c) $\gamma_{\mathrm{SE}_{\mathbf{I}, k}}^{2}(\mathbf{I})$

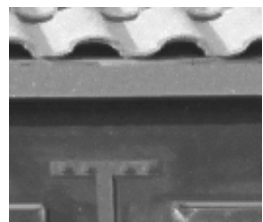

(f) $\varphi_{\mathrm{SE}_{\mathbf{I}, k}}^{2}(\mathbf{I})$

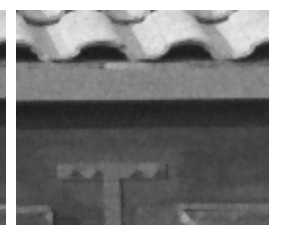

(d) $\gamma_{\mathrm{SE}}^{2}(\mathbf{I})$

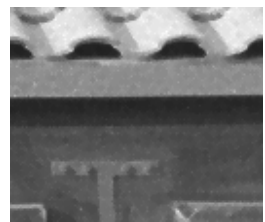

(g) $\varphi_{\mathrm{SE}}^{2}(\mathbf{I})$

Fig. 5. House pattern example is a $110 \times 130$ image. Patches are squares of $5 \times 5$, $\sigma=200$ and $k=5$. The $k$-graph contains only one connected component.

\section{References}

1. A. Baudes, B.C., Morel, J.: A review of image denoising algorithms with a new one. Multiscale Modeling and Simulation 4(2), 490-530 (2005)

2. Angulo, J., Velasco-Forero, S.: Structurally adaptive math. morph. based on nonlinear scale-space decomp. Image Analysis \& Stereology 30(2), 111-122 (2011)

3. Angulo, J.: Morphological bilateral filtering and spatially-variant adaptive structuring functions. In: ISMM'11, LNCS, vol. 6671, pp. 212-223. Springer (2011)

4. Angulo, J., Velasco-Forero, S.: Stochastic morphological filtering and bellmanmaslov chains. In: Proceeding of ISMM'13 (2013)

5. Beucher, S., Blosseville, J.M., Lenoir, F.: Traffic spatial measurements using video image processing. In: Proc. Intelligent Robots and Computer Vision. SPIE (1988)

6. Bouaynaya, N., Charif-Chefchaouni, M., Schonfeld, D.: Theoretical Foundations of Spatially-Variant Mathematical Morphology Part I: Binary Images. IEEE Trans. Patt. Ana. Mach. Lear. 30(5), 823-836 (2008)

7. Bresson, X., Chan, T.F.: Non-local unsupervised variational image segmentation models. Tech. rep., UCLA CAM (2008)

8. Buades, A., Coll, B., Morel, J.M.: Image denoising methods. a new nonlocal principle. SIAM Review 52(1), 113-147 (Feb 2010)

9. Burgeth, B., Weickert, J.: An explanation for the logarithmic connection between linear and morph. system theory. Int. J. Comp. Vision 64(2-3), 157-169 (2005)

10. Cuisenaire, O.: Locally adaptable mathematical morphology using distance transformations. Pattern Recognition 39(3), 405-416 (Mar 2006) 


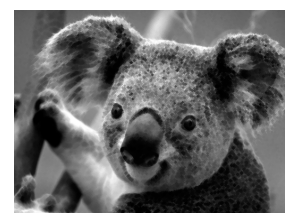

(a) $\varepsilon_{\mathrm{SE}}^{2}(\mathbf{I})$

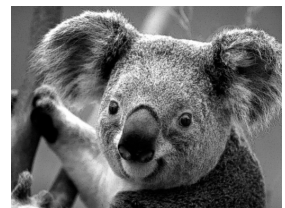

(e) $\varepsilon_{\mathrm{SE}_{\mathbf{I}, k}}^{2}(\mathbf{I})$

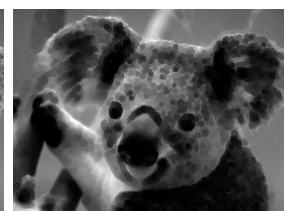

(b) $\varepsilon_{\mathrm{SE}}^{5}(\mathbf{I})$

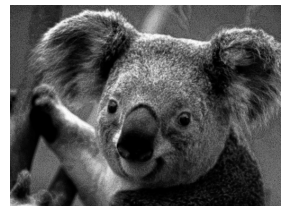

(f) $\varepsilon_{\mathrm{SE}_{\mathbf{I}, k}}^{5}(\mathbf{I})$

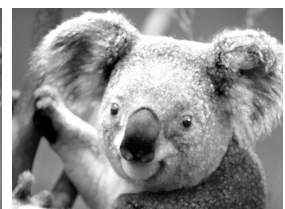

(c) $\delta_{\mathrm{SE}}^{2}(\mathbf{I})$

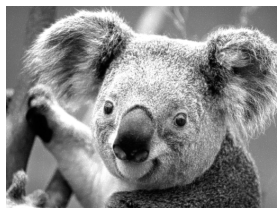

(g) $\delta_{\mathrm{SE}_{\mathbf{I}, k}}^{2}(\mathbf{I})$

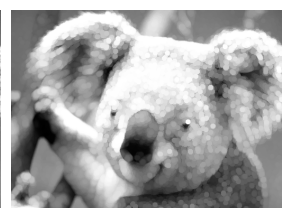

(d) $\delta_{\mathrm{SE}}^{5}(\mathbf{I})$

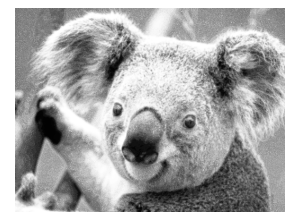

(h) $\delta_{\mathrm{SE}_{\mathbf{I}, k}}^{5}(\mathbf{I})$

Fig. 6. Examples of classical (top) and sparse nonlocal (bottom) erosion and dilations, where patches are squares $5 \times 5, \sigma=200$ and $k=5$. Original image in Fig. 2 .

11. Debayle, J., Pinoli, J.C.: Spatially adaptive morphological image filtering using intrinsic structuring elements. Image Analysis and Stereology 39(3), 145-158 (2005)

12. Gilboa, G., Osher, S.: Nonlocal Linear Image Regularization and Supervised Segmentation. Multiscale Modeling \& Simulation 6(2), 595-630 (2007)

13. Grazzini, J., Soille, P.: Edge-preserving smoothing using a similarity measure in adaptive geodesic neighbourhoods. Pattern Recognition 42(10), 2306 - 2316 (2009)

14. Heijmans, H.: Theoretical aspects of gray-level morphology. IEEE Trans. Patt. Ana. Mach. Lear. 13(6), 568-582 (1991)

15. Katkovnik, V., Foi, A., Egiazarian, K., Astola, J.: From local kernel to nonlocal multiple-model image denoising. Inte.Journal of Comp. Vision 86, 1-32 (2010)

16. Lerallut, R., Decencière, E., Meyer, F.: Image filtering using morphological amoebas. Image and Vision Computing 4(25), 395-404 (2007)

17. Lézoray, O., Elmoataz, A.: Nonlocal and multivariate mathematical morphology. In: International Conference on Image Processing (IEEE) (2012)

18. Maragos, P.: Slope transforms: theory and application to nonlinear signal processing. IEEE Transactions on Signal Processing 43(4), 864-877 (1995)

19. Milanfar, P.: Symmetrizing smoothing filters. SIAM Journal on Imaging Sciences (2013), to appear

20. Morard, V., Decencière, E., Dokládal, P.: Region growing structuring elements and new operators based on their shape. In: Signal and Image Proc. ACTA Press (2011)

21. Roerdink, J.B.T.M.: Adaptivity and group invariance in mathematical morphology. In: ICIP. pp. 2253-2256 (2009)

22. Salembier, P.: Study on nonlocal morph. operators. In: ICIP. pp. 2269-2272 (2009)

23. Serra, J.: Image Analysis and Mathematical Morphology. Academic Press, Inc., Orlando, FL, USA (1982)

24. Serra, J.: Image Analysis and Mathematical Morphology Volume 2: Theoretical Advances. Academic Press (1988)

25. Ta, V., Elmoataz, A., Lezoray, O.: Nonlocal PDEs-based morphology on weighted graphs for image and data proc. IEEE Trans. Im. Proc. 20(6), 1504-1516 (2011) 